\title{
Prevention of Chemotherapy-Induced Anemia and Thrombocytopenia by Constant Administration of Stem Cell Factor
}

Monica Bartucci ${ }^{1}$, Rosanna Dattilo ${ }^{1}$, Daniela Martinetti ${ }^{3}$, Matilde Todaro ${ }^{4}$, Giuseppina Zapparelli ${ }^{1}$, Antonio Di Virgilio ${ }^{2}$, Mauro Biffoni ${ }^{1}$, Ruggero De Maria ${ }^{1}$, and Ann Zeuner ${ }^{1}$

\section{Abstract}

Purpose: Chemotherapy-induced apoptosis of immature hematopoietic cells is a major cause of anemia and thrombocytopenia in cancer patients. Although hematopoietic growth factors such as erythropoietin and colony-stimulating factors cannot prevent the occurrence of drug-induced myelosuppression, stem cell factor (SCF) has been previously shown to protect immature erythroid and megakaryocytic cells in vitro from drug-induced apoptosis. However, the effect of SCF in vivo as a single myeloprotective agent has never been elucidated.

Experimental Design: The ability of SCF to prevent the occurrence of chemotherapy-induced anemia and thrombocytopenia was tested in a mouse model of cisplatin-induced myelosuppression. To highlight the importance of maintaining a continuous antiapoptotic signal in immature hematopoietic cells, we compared two treatment schedules: in the first schedule, SCF administration was interrupted during chemotherapy treatment and resumed thereafter, whereas in the second schedule, SCF was administered without interruption for 7 days, including the day of chemotherapy treatment.

Results: The administration of SCF to cisplatin-treated mice could preserve bone marrow integrity, inhibit apoptosis of erythroid and megakaryocytic precursors, prevent chemotherapy-induced anemia, and rapidly restore normal platelet production. Treatment with SCF increased the frequency of Bcl-2/Bcl-XLpositive bone marrow erythroid cells and sustained Akt activation in megakaryocytes. Myeloprotection was observed only when SCF was administered concomitantly with cisplatin and kept constantly present during the days following chemotherapy treatment.

Conclusions: SCF treatment can prevent the occurrence of chemotherapy-induced anemia and thrombocytopenia in mice, indicating a potential use of this cytokine in the supportive therapy of cancer patients. Clin Cancer Res; 17(19); 6185-91. (C2011 AACR.

\section{Introduction}

Chemotherapy-induced bone marrow damage results in anemia and thrombocytopenia that threaten the patients' quality of life and the overall efficacy of anticancer treatments. Hematopoietic growth factors such as erythropoi-

Authors' Affiliations: ${ }^{1}$ Department of Hematology, Oncology and Molecular Medicine, and ${ }^{2}$ Service of Biotechnology and Animal Welfare, Istituto Superiore di Sanità, Rome; ${ }^{3}$ Istituto Oncologico del Mediterraneo, Viagrande, Catania; and ${ }^{4}$ Department of Surgical and Oncological Sciences, University of Palermo, Palermo, Italy

Note: Supplementary data for this article are available at Clinical Cancer Research Online (http://clincancerres.aacrjournals.org/).

R. Dattilo and D. Martinetti contributed equally to this work.

Corresponding Author: Ann Zeuner, Department of Hematology, Oncology and Molecular Medicine, Istituto Superiore di Sanità, Viale Regina Elena 299, Rome, Italy. Phone: 39-06-4990-2479; Fax: 39-06-4938-7087; E-mail: a.zeuner@iss.it

doi: 10.1158/1078-0432.CCR-11-1232

C2011 American Association for Cancer Research. etin and colony-stimulating factors are commonly used to promote hematopoietic recovery following chemotherapy, but they cannot prevent the occurrence of drug-induced myelosuppression. Stem cell factor (SCF) is produced by stromal cells of the bone marrow and binds the receptor c-kit expressed on hematopoietic stem and progenitor cells (1). It is essential for erythroid homeostasis, as mice defective for SCF or its receptor c-kit display severe macrocytic anemia and inefficient response to stress erythropoiesis (2-7). SCF administration has been shown to stimulate hematopoiesis in rodents, primates, and humans by increasing the number of bone marrow stem and progenitor cells $(8-10)$ and promote recovery after cytotoxic damage (11). Besides stimulating hematopoietic cell expansion, SCF is a potent antiapoptotic factor for erythroid and megakaryocytic cells (12-15). In vitro, SCF can prevent chemotherapy-induced apoptosis of immature erythroblasts and megakaryocytes, which are exquisitely vulnerable to cytotoxic agents $(14,15)$. In this report, we show that SCF acts as a myeloprotective agent in vivo, being able 


\section{Translational Relevance}

Chemotherapy-induced anemia and thrombocytopenia occur in a large portion of patients receiving myelosuppressive chemotherapy, leading to therapy-related complications and treatment delay/reduction/discontinuation that negatively influence patient survival. The development of effective supportive strategies for the treatment of chemotherapy-induced myelosuppression is therefore essential to improve the outcome of both conventional and targeted antineoplastic therapies.

Stem cell factor (SCF) is mainly used in second-line treatment regimens for mobilization-refractory patients. Here, we show that SCF protects bone marrow from chemotherapy-induced damage and prevents the occurrence of anemia and thrombocytopenia in the peripheral blood of treated mice. We also show for the first time that the efficacy of SCF as a myeloprotective agent depends on the schedule of administration.

The observation that SCF can protect erythroid and megakaryocytic precursors in vivo provides a rationale for a future use of this cytokine in preventing druginduced anemia and thrombocytopenia in cancer patients.

to protect bone marrow cells from cisplatin-induced damage. In particular, SCF protected bone marrow erythroid $\left(\right.$ TER $\left.119^{+}\right)$and megakaryocytic $\left(\mathrm{CD} 41^{+}\right)$precursors from chemotherapy-induced depletion and prevented the occurrence of anemia and thrombocytopenia in the peripheral blood of chemotherapy-treated mice. Importantly, only an administration schedule that maintained a constant presence of SCF during and after chemotherapy treatment could prevent bone marrow damage and consequent anemia/thrombocytopenia, thus indicating the need for an uninterrupted delivery of survival stimuli to achieve effective myeloprotection.

\section{Materials and Methods}

\section{Mice treatment}

Animal experiments were conducted according to the National Animal Experimentation Guidelines (D.L.116/ 92) upon approval of the experimental protocol by the Institutional Animal Experimentation Committee. Sixweek-old C57/BL6 female mice weighing approximately $20 \mathrm{~g}$ were purchased from Jackson Laboratories and maintained with food and water ad libitum for the duration of the studies. Mice were treated as follows: the "Control" group received matched PBS injections, the "Cisplatin" group received a single intraperitoneal dose of $7.5 \mathrm{mg} / \mathrm{kg}$ cisplatin (Sigma Aldrich) at day 0, the "SCF" group received $50 \mu \mathrm{g} / \mathrm{kg}$ recombinant murine SCF (mSCF; Peprotech) dissolved in PBS twice a day from days -1 to 7 , "Interrupted" mice received $7.5 \mathrm{mg} / \mathrm{kg}$ cisplatin at day 0 and 100 $\mu \mathrm{g} / \mathrm{kg} \mathrm{mSCF}$ at day -1 and once a day from days 1 to 7 (being SCF treatment interrupted during cisplatin admin- istration), and "Constant" mice received cisplatin at day 0 and $50 \mu \mathrm{g} / \mathrm{kg} \mathrm{mSCF}$ twice a day from days 0 to 7 (covering also the day of cisplatin administration). SCF was administered subcutaneously.

\section{Microscopy and flow cytometry}

Bone marrow sections were prepared as follows: At the end of the treatment, mice were sacrificed and femurs were removed, fixed in buffered paraformaldehyde 10\% for 24 hours, washed, and exposed to decalcifying solution (0.05 mol/L EDTA, 5N NaOH). Samples were deparaffinized and hydrated, and staining reactions were then carried out on $6-\mu \mathrm{m}$-thick sections. To evaluate bone marrow cellularity, histologic sections were stained with hematoxylin/eosin. Terminal deoxynucleotidyl transferase-mediated dUTP nick end labeling (TUNEL) staining was carried out with the In Situ Cell Death Detection Kit (Roche Molecular Biochemicals) according to the manufacturer's instructions. For immunofluorescence analysis and MayGrünwald-Giemsa staining, mice were sacrificed at the end of the treatment, femurs were harvested, and marrow flushed with a $23 \mathrm{G}(0.45 \times 10 \mathrm{~mm})$ syringe needle to collect single-cell suspensions. For immunofluorescence analysis of mouse bone marrow cells double positive for CD41/phospho-Akt, TER119/Bcl-2, or TER119/Bcl-XL, bone marrow cells were stained with phycoerythrin (PE)-conjugated anti-CD41 (BD Pharmingen) or antiTER119 (eBioscience) and sorted with a FACSaria flow cytometer (Becton Dickinson). Sorted cells were then stained with primary antibodies against phospho-Akt (Cell Signaling), Bcl-2 (clone C-21), or Bcl-XL (clone H5; Santa Cruz Biotechnology). Alexa-Fluor 488 secondary antibody was from Invitrogen Molecular Probes. Bone marrow sections and May-Grünwald-Giemsa-stained cells were analyzed with a Nikon Eclipse E1000 microscope equipped with PlanFluor $40 \times$ dry objectives (numerical aperture: 0.75 ) and PlanApo $60 \times$ oil objectives (numerical aperture: 1.4), respectively (Nikon). Images were taken with a Nikon DXM1200 RGB camera and the Nikon ACT-1 Software. Immunofluorescence images were taken with an FV1000 confocal microscope (Olympus) equipped with a $60 \times$ oil immersion objective and the Olympus Fluoview software.

To evaluate $\mathrm{CD} 41^{+}$and TER $119^{+}$bone marrow populations by flow cytometry, cells were harvested as described earlier and stained with PE-conjugated antiCD41 or fluorescein isothiocyanate-conjugated antiTER119 (eBioscience). Samples were analyzed with a FACSCanto flow cytometer equipped with FACSDiva software.

\section{Peripheral blood analyses}

Peripheral blood was obtained from retro-orbital bleeding of mice treated as described earlier. Bleeding was carried out at day 10 for hemoglobin analysis and at days $2+10$ for platelet analysis. Blood was dripped directly after removal into tubes containing $0.5 \mathrm{~mol} / \mathrm{L}$ EDTA. The analysis of peripheral blood parameters was conducted by a contract laboratory (AppiaLab) within 2 hours from 


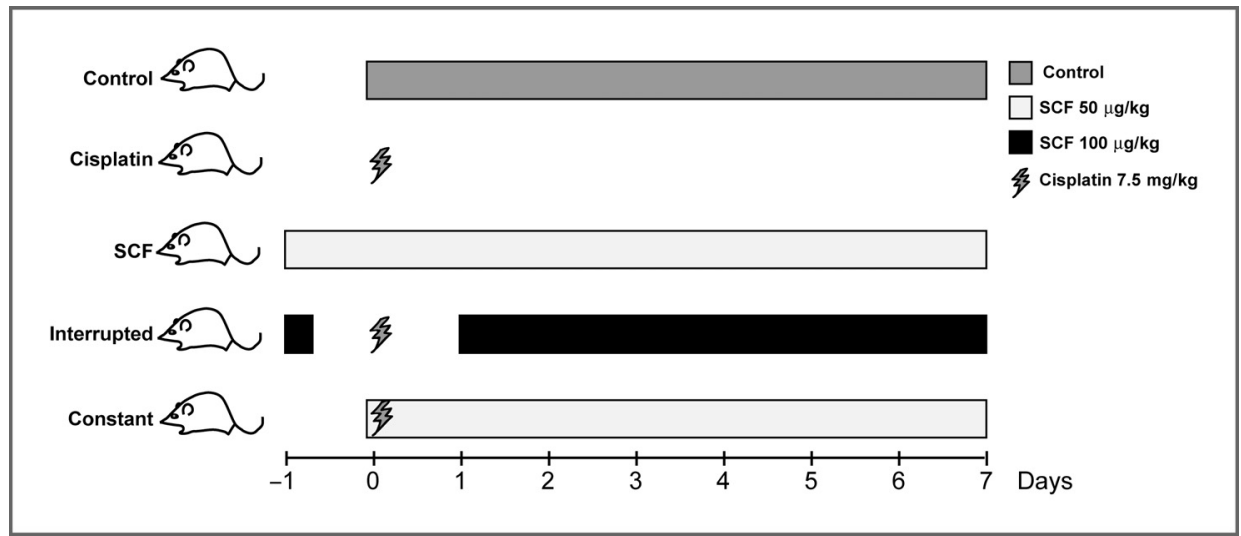

Figure 1. Representation of the treatment schedule with SCF and cisplatin. Five groups of mice were treated as follows: the Control group received matched PBS injections, the Cisplatin group received a single intraperitoneal dose of $7.5 \mathrm{mg} / \mathrm{kg}$ cisplatin at day 0 (bolt), the SCF group subcutaneously received $50 \mu \mathrm{g} / \mathrm{kg}$ recombinant $\mathrm{mSCF}$ twice a day from days -1 to 7 , the Interrupted group received $7.5 \mathrm{mg} / \mathrm{kg}$ cisplatin at day 0 (bolt) and $100 \mu \mathrm{g} / \mathrm{kg} \mathrm{mSCF}$ at day -1 and once a day from days 1 to 7 , and the Constant group received cisplatin at day 0 (bolt) and $50 \mu \mathrm{g} / \mathrm{kg} \mathrm{mSCF}$ twice a day from days 0 to 7 .

bleeding. For hemoglobin and platelet evaluation, mice received 2 injections of $5 \mathrm{mg} / \mathrm{kg}$ cisplatin at days 0 and 4 , which was necessary to produce a constant decrease in platelet number, avoiding rebound effects that occur after a single chemotherapy treatment (16).

\section{Statistical analysis}

The statistical significance of results was calculated using GraphPad Prism 4 (GraphPad Software Inc.; www. graphpad.com) and analyzed by means of one-way ANOVA and Bonferroni's multiple comparison tests. A

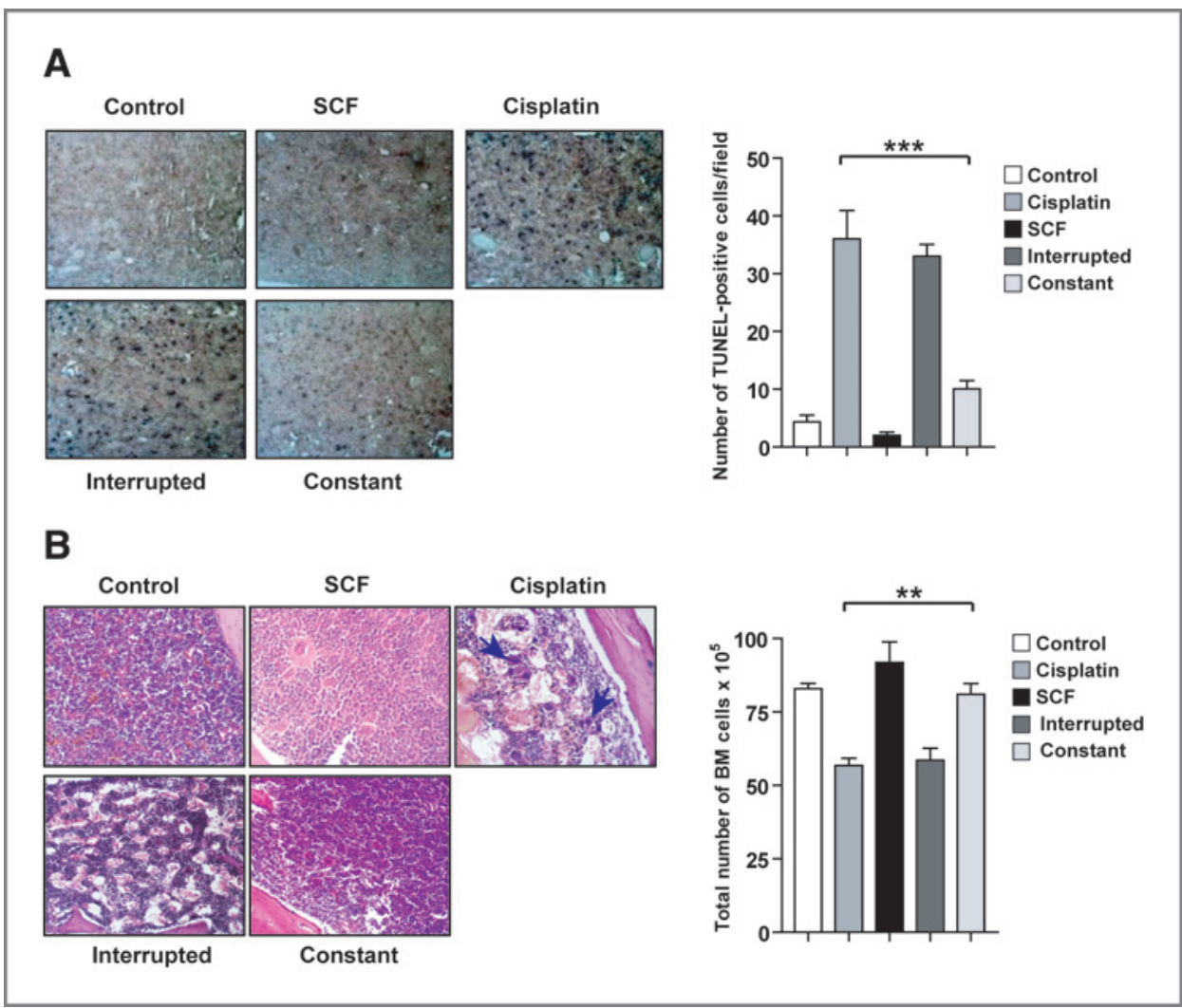

Figure 2. Constant administration of SCF protects bone marrow from chemotherapy-induced damage. Mice were treated as described in Figure 1. A, TUNEL staining of femur sections obtained at day 4 from the 5 groups of mice described earlier (left) and the number of TUNEL-positive cells assessed in 3 independent experiments carried out with 4 mice for each group (right). Images were taken with 40× magnification. One-way ANOVA and Bonferroni's multiple comparison tests showed a statistical significance of ${ }^{* \star}, P<0.001$ between Cisplatin and Constant group mice. B, hematoxylin/eosin staining of femur sections derived at day 7 from the 5 groups of mice described earlier (left) and absolute numbers of bone marrow (BM) cells extracted from contralateral femurs and counted on a Bürker counting chamber (right). Images were taken with $40 \times$ magnification. Arrows on the Cisplatin panels indicate clusters of dysplastic megakaryocytes. The experiment was repeated 4 times with 4 mice for each group. The comparison between Cisplatin and Constant group mice with one-way ANOVA and Bonferroni's multiple comparison tests showed a statistical significance of ${ }^{*}, P<0.01$. 


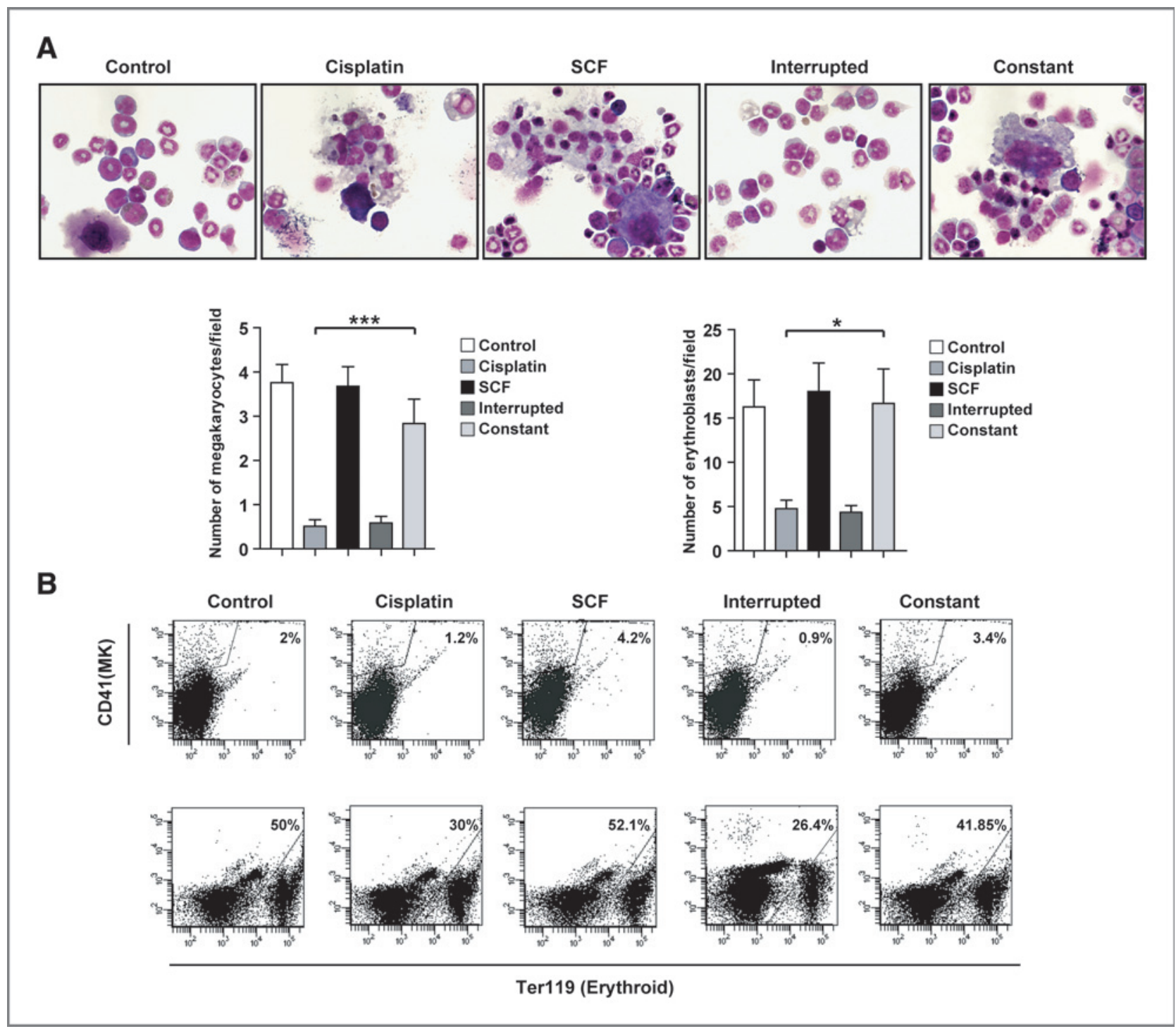

Figure 3. SCF specifically protects erythroid and megakaryocytic precursors from chemotherapy-induced depletion. A, May-Grünwald-Giemsa staining of bone marrow cells extracted from the femurs of mice treated as described earlier (top) and numbers of megakaryocytes (bottom, left) and erythroblasts (bottom, right) counted on May-Grünwald-Giemsa-stained slides derived from 4 independent experiments. Images were taken with $60 \times$ magnification. The difference in megakaryocyte numbers according to one-way ANOVA and Bonferroni's multiple comparison tests was statistically significant with ${ }^{\star \star}, P<0.001$ between Cisplatin and Constant group mice. The difference in erythroblast numbers according to one-way ANOVA and Bonferroni's multiple comparison tests was statistically significant with ${ }^{*}, P<0.05$ between Cisplatin and Constant group mice. B, flow cytometric analysis of CD41 (top; MK, megakaryocytes) and TER119 expression (bottom; Erythroid, erythroid precursors) in bone marrow cells extracted at day 7 from C57/BL6 mice treated as described earlier. The panels show a representative set of results obtained from 2 independent experiments carried out with 6 mice for each group.

value of $P<0.05$ is represented by a single asterisk, $P<0.01$ is indicated by a double asterisk, and $P<0.001$ is indicated by 3 asterisks.

\section{Results and Discussion}

To investigate whether SCF could protect bone marrow cells from chemotherapy-induced destruction in vivo, we induced myelosuppression in mice with a single dose of cisplatin and administered SCF according to 2 treatment schedules. In the cohort of mice indicated as Interrupted, SCF was administered 24 hours before and 24 hours after cisplatin injection and once daily for 6 additional days. In the cohort of mice indicated as Constant, SCF was administered 4 hours before and 4 hours after cisplatin injection and then twice a day for 6 additional days. In the Constant group, the schedule of administration ensured a constant availability of SCF throughout the treatment period, considering that SCF has an elimination half-life of approximately 8 hours (17), whereas in the Interrupted 
Figure 4. SCF activates antiapoptotic pathways in erythroid and megakaryocytic cells in vivo. Immunofluorescence analysis of $\mathrm{Bcl}-2, \mathrm{Bcl}-\mathrm{XL}$, and phospho-Akt (pAkt) in bone marrow cells extracted from mice treated as described in Figure 1. Cells were sorted for the expression of TER119 (Erythroid, erythroid precursors) or CD41 (MK, megakaryocytes) and then stained with anti-Bcl-2, anti-Bcl$\mathrm{XL}$, or anti-phospho-Akt, respectively. Images were taken with an Olympus FV1000 confocal microscope with $60 x$

magnification and $6 \times$ zoom. The experiment was repeated twice with 6 mice for each group.

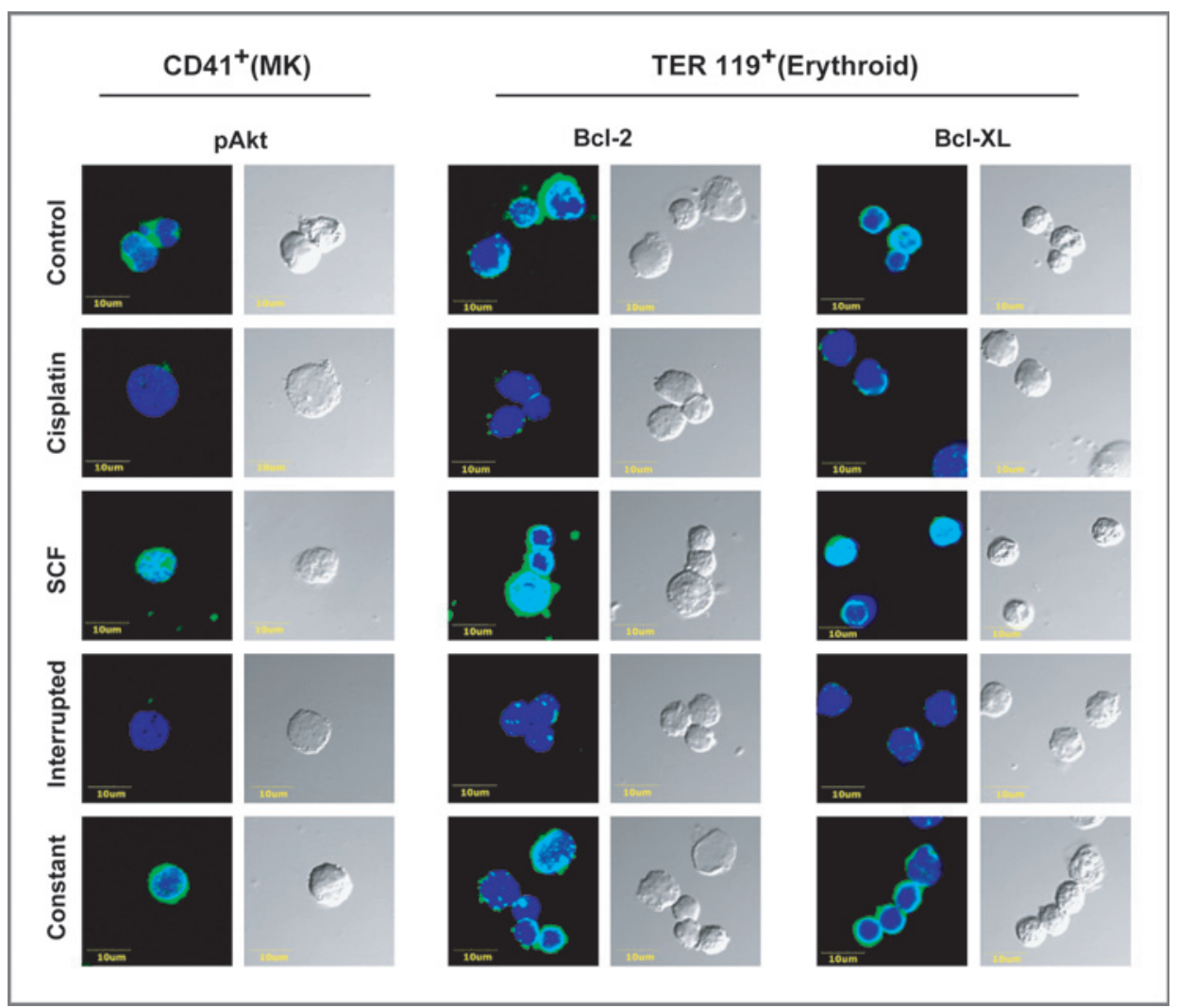

group, this condition was intentionally not fulfilled (Fig. 1).

First, we analyzed bone marrow sections from control mice (treated with PBS or with SCF alone) and mice treated with cisplatin (in the presence or absence of SCF delivered, according to the schedules described earlier) stained with TUNEL to reveal the presence of apoptotic cells or with hematoxylin/eosin to display bone marrow structure. TUNEL-stained bone marrow sections showed a high number of apoptotic cells both in mice treated with cisplatin alone ( $36 \pm 6$ per field) and in Interrupted mice ( $33 \pm 3$ per field), whereas in Constant mice, the number of dying cells was significantly lower $(9 \pm 2$ per field; $P<0.001$; Fig. 2 A). Upon hematoxylin/eosin staining, the bone marrow of cisplatin-treated mice showed a strong hypoplasia with myelofibrosis and clusters of dysplastic megakaryocytes (Fig. 2B, left, arrows). The bone marrow of Interrupted mice was similar to that of mice treated with cisplatin alone, indicating that an intermittent administration of SCF compromises the ability of this cytokine to prevent bone marrow destruction (Fig. 2B, left). These observations were confirmed by counting the absolute number of cells extracted from mouse femurs (Fig. 2B, right). In contrast, bone marrow structure and cell numbers of Constant mice resembled those of mice treated with PBS, indicating that constant administration of SCF protects bone marrow cells from chemotherapy-induced damage. The analysis of MayGrünwald-Giemsa-stained cells extracted from femurs of mice treated as described earlier showed that megakaryocytes and erythroblasts were significantly more abundant in Constant mice than in mice treated with cisplatin alone (Fig. 3A; $P<0.001$ and $P<0.05$, respectively).

An analysis of bone marrow cells of the granulocytic lineage on May-Grünwald-Giemsa-stained slides revealed a significant decrease of granulocytic precursors in the Cisplatin and the Interrupted samples, whereas SCF treatment restored the levels of immature granulocytes in the Constant group (data not shown). However, a massive presence of mature granulocytes in the Cisplatin and the Interrupted samples (likely represented by the long-lived bone marrow reservoir; ref. 18) rendered difficult to prove a protective effect of SCF toward the granulocytic lineage. The relative abundance of megakaryocytic and erythroid cells was confirmed by fluorescence-activated cell-sorting analysis of $\mathrm{CD} 41^{+}$and TER $119^{+}$cells in the bone marrow of control and treated mice, respectively (Fig. $3 \mathrm{~B}$ and Supplementary Fig. S1).

SCF was previously shown to prevent chemotherapyinduced apoptosis of purified human erythroblasts and megakaryocytes (which were shown to represent the preferential target of drug-induced damage within the erythroid and megakaryocytic lineage, respectively) by inducing an increase in antiapoptotic factors. Specifically, SCF upregulates $\mathrm{Bcl}-2 / \mathrm{Bcl}-\mathrm{XL}$ in erythroid precursors, whereas in megakaryocytes, it increases Akt activation with subsequent Bad phosphorylation $(14,15)$. To investigate 
whether such antiapoptotic mechanisms were activated in murine hematopoietic precursors upon SCF treatment in vivo, we sorted cells positive for TER119 (erythroid precursors) or CD41 (megakaryocytic progenitors and megakaryocytes) from the bone marrow of mice treated with vehicle, cisplatin, SCF only, or cisplatin plus SCF in the Interrupted or Constant modality. Subsequent staining of sorted bone marrow cells with anti-phosphorylated Akt (for the $\mathrm{CD} 41^{+}$population) or with $\mathrm{Bcl}-2$ or anti-Bcl-XL (for the TER $119^{+}$population) showed an increased frequency of Akt phosphorylation in $\mathrm{CD} 41^{+}$megakaryocytic cells of Constant mice as compared with mice treated with cisplatin alone (Fig. 4). Similarly, an increased number of TER $119^{+}$bone marrow erythroid cells expressed Bcl-2 and Bcl-XL in Constant mice as compared with mice treated with cisplatin alone (Fig. 4). In both cases, Interrupted mice did not differ significantly from mice treated with cisplatin alone. These results indicate that only a continuous stimulation with SCF can elicit antiapoptotic signals that act through the mitochondrial pathway, thus preventing the depletion of bone marrow erythroid and megakaryocytic cells.

Then, we hoped to investigate the consequences of SCFinduced myeloprotection on peripheral blood red cells and platelets. To obtain a sustained decrease of hemoglobin and platelets in the peripheral blood, mice were subjected to 2 injections of cisplatin at days 0 and 4 of treatment, respectively. Subsequent peripheral blood analyses revealed that at day 10, hemoglobin levels of Constant mice were significantly higher than those of cisplatin-treated animals $(P<0.01)$ and similar to those of vehicletreated controls, indicating that SCF efficiently prevented the occurrence of cisplatin-induced anemia (Fig. 5A). An assessment of platelet levels showed a progressive decrease of platelet levels in the Cisplatin group and, to a lesser extent, in the Interrupted group (Fig. 5B). Conversely, platelet levels of the Constant group started to increase after an initial reduction and, 10 days after the first chemotherapy insult, reached the levels detected in control animals (Fig. 5B). Altogether, these results indicate that a constant administration of SCF can prevent the occurrence of chemotherapy-induced anemia and thrombocytopenia through a specific protection of immature erythroid and megakaryocytic cells. Investigations are ongoing in our laboratory to determine whether the protective effects of SCF on the bone marrow may involve other immature hematopoietic cells such as CD $34^{+}$hematopoietic progenitors. A comprehensive analysis of pathways activated by SCF in immature hematopoietic cells conducted by reverse-phase proteomic arrays shows activation of multiple antiapoptotic pathways besides Bcl-2/Bcl-XL increase and Akt phosphorylation (F. Pedini and A. Zeuner, unpublished data), suggesting that a network of signals activated by c-kit contributes to SCF-mediated hematopoietic protection. The ability of SCF to activate an extensive network of survival signals may explain the multilineage antiapoptotic activity of this cytokine, which would provide an advantage over more restricted

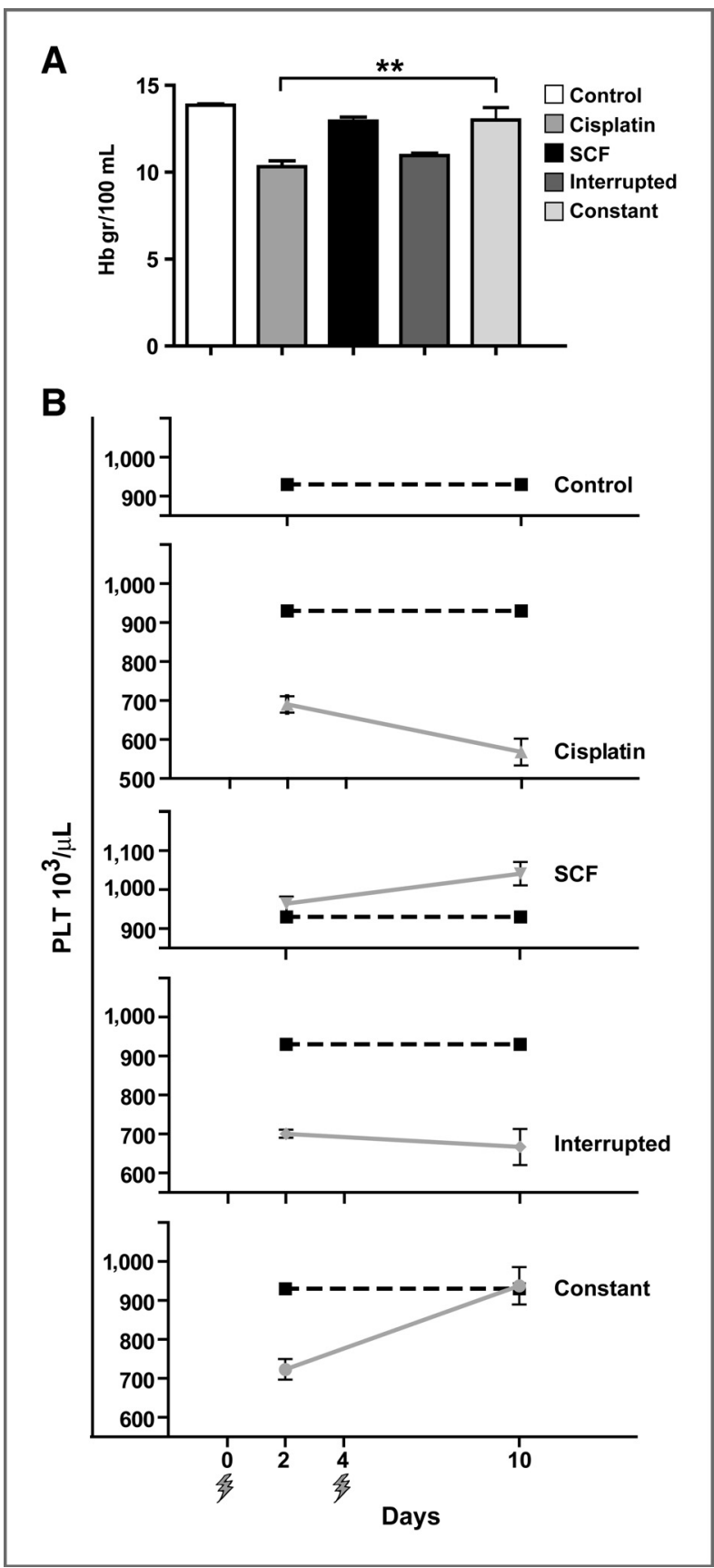

Figure 5. SCF prevents chemotherapy-induced decrease of hemoglobin and platelets in the peripheral blood. A, hemoglobin $(\mathrm{Hb})$ levels in the peripheral blood of mice treated with SCF as described in Figure 1 and with $5 \mathrm{mg} / \mathrm{kg}$ cisplatin at days 0 and 4 . The blood was withdrawn at day 10 by retro-orbital bleeding and analyzed with an automated analyzer. The experiment was repeated 6 times with groups of 4 mice each. One-way ANOVA analysis with Bonferroni's multiple comparison tests showed a statistical significance of ${ }^{* *}, P<0.01$ between Cisplatin and Constant group mice. B, platelet (PLT) numbers in the peripheral blood of mice treated as in A. Mice belonging to the 5 treatment groups were treated with $5 \mathrm{mg} / \mathrm{kg}$ cisplatin at days 0 and 4 (bolt). Blood was obtained by retro-orbital bleeding at days 2 and 10 and analyzed with an automated analyzer. The experiment was repeated 6 times with groups of 4 mice each. 
growth factors (erythropoiesis- and granulopoiesisstimulating agents) currently used in the supportive therapy of cancer patients. Although further in vivo experiments with non-human primates would be useful to confirm the myeloprotective activity of SCF, this cytokine may find a future use to prevent drug-induced anemia and thrombocytopenia in cancer patients with tumors that do not rely on c-kit signaling for growth and survival.

\section{Disclosure of Potential Conflicts of Interest}

No potential conflicts of interest were disclosed.

\section{References}

1. Broudy VC. Stem cell factor and hematopoiesis. Blood 1997;90:134564.

2. Zsebo KM, Williams DA, Geissler EN, Broudy VC, Martin FH, Atkins $\mathrm{HL}$, et al. Stem cell factor is encoded at the $\mathrm{SI}$ locus of the mouse and is the ligand for the c-kit tyrosine kinase receptor. Cell 1990;63: 213-24.

3. Huang E, Nocka K, Beier DR, Chu TY, Buck J, Lahm HW, et al. The hematopoietic growth factor $\mathrm{KL}$ is encoded by the $\mathrm{SI}$ locus and is the ligand of the c-kit receptor, the gene product of the W locus. Cell 1990;63:225-33.

4. Copeland NG, Gilbert DJ, Cho BC, Donovan PJ, Jenkins NA, Cosman $\mathrm{D}$, et al. Mast cell growth factor maps near the steel locus on mouse chromosome 10 and is deleted in a number of steel alleles. Cell 1990;63:175-83.

5. Perry JM, Harandi OF, Paulson RF. BMP4, SCF, and hypoxia cooperatively regulate the expansion of murine stress erythroid progenitors. Blood 2007;109:4494-502.

6. Chabot B, Stephenson DA, Chapman VM, Besmer P, Bernstein A. The proto-oncogene c-kit encoding a transmembrane tyrosine kinase receptor maps to the mouse W locus. Nature 1988;335:88-9.

7. Geissler EN, Ryan MA, Housman DE. The dominant-white spotting (W) locus of the mouse encodes the c-kit proto-oncogene. Cell 1988; 55:185-92.

8. Molineux G, Migdalska A, Szmitkowski M, Zsebo K, Dexter TM. The effects on hematopoiesis of recombinant stem cell factor (ligand for c-kit) administered in vivo to mice either alone or in combination with granulocyte colony-stimulating factor. Blood 1991;78:961-6.

9. Andrews RG, Knitter GH, Bartelmez SH, Langley KE, Farrar D, Hendren RW, et al. Recombinant human stem cell factor, a c-kit ligand, stimulates hematopoiesis in primates. Blood 1991;78:1975-80.

\section{Acknowledgments}

We thank Dr. Paola Cosentino (Appialab center for veterinary analysis, Rome) for discussion and support, Giuseppe Loreto for graphics, and Stefano Guida for technical assistance.

\section{Grant Support}

This work was supported by the Italian Association for Cancer Research (AIRC; Investigator grant 5717 to A. Zeuner and fellowship to G. Zapparelli). The costs of publication of this article were defrayed in part by the payment of page charges. This article must therefore be hereby marked advertisement in accordance with 18 U.S.C. Section 1734 solely to indicate this fact.

Received May 12, 2011; revised July 19, 2011; accepted August 9, 2011; published OnlineFirst August 25, 2011

10. Tong J, Gordon MS, Srour EF, Cooper RJ, Orazi A, McNiece I, et al. In vivo administration of recombinant methionyl human stem cell factor expands the number of human marrow hematopoietic stem cells. Blood 1993;82:784-91.

11. Blaise D, Faucher C, Vey N, Caraux J, Maraninchi D, Chabannon C. Rescue of haemopoiesis by a combination of growth factors including stem-cell factor. Lancet 2000;356:1325-6.

12. Sui $X$, Krantz SB, Zhao ZJ. Stem cell factor and erythropoietin inhibit apoptosis of human erythroid progenitor cells through different signalling pathways. Br J Haematol 2000;110:63-70.

13. Endo T, Odb A, Satoh I, Haseyama Y, Nishio M, Koizumi K, et al. Stem cell factor protects $\mathrm{c}-\mathrm{kit}^{+}$human primary erythroid cells from apoptosis. Exp Hematol 2001;29:833-41.

14. Zeuner A, Pedini F, Signore M, Testa U, Pelosi E, Peschle C, et al. Stem cell factor protects erythroid precursor cells from chemotherapeutic agents via up-regulation of BCL-2 family proteins. Blood 2003;102:87-93.

15. Zeuner A, Signore M, Martinetti D, Bartucci M, Peschle C, De Maria R, et al. Chemotherapy-induced thrombocytopenia derives from the selective death of megakaryocyte progenitors and can be rescued by stem cell factor. Cancer Res 2007;67:4767-73.

16. Hunt $P$, Zsebo KM, Hokom MM, Hornkohl A, Birkett NC, del Castillo $\mathrm{JC}$, et al. Evidence that stem cell factor is involved in the rebound thrombocytosis that follows 5-fluorouracil treatment. Blood 1992; 80:904-11.

17. Lynch DH, Jacobs C, DuPont D, Eisenman J, Foxworthe D, Martin U, et al. Pharmacokinetic parameters of recombinant mast cell growth factor (rMGF). Lymphokine Cytokine Res 1992;11:233-43.

18. Boxio R, Bossenmeyer-Pourie C, Steinckwich N, Dournon C, Nusse $\mathrm{O}$. Mouse bone marrow contains large numbers of functionally competent neutrophils. J Leukoc Biol 2004;75:604-11. 


\section{Clinical Cancer Research}

\section{Prevention of Chemotherapy-Induced Anemia and Thrombocytopenia by Constant Administration of Stem Cell Factor}

Monica Bartucci, Rosanna Dattilo, Daniela Martinetti, et al.

Clin Cancer Res 2011;17:6185-6191. Published OnlineFirst August 25, 2011.

Updated version Access the most recent version of this article at: doi:10.1158/1078-0432.CCR-11-1232

Supplementary Access the most recent supplemental material at:

Material http://clincancerres.aacrjournals.org/content/suppl/2011/08/25/1078-0432.CCR-11-1232.DC1.html

Cited Articles This article cites by 18 articles, 9 of which you can access for free at:

http://clincancerres. aacrjournals.org/content/17/19/6185.full.html\#ref-list-1

E-mail alerts Sign up to receive free email-alerts related to this article or journal.

Reprints and Subscriptions

To order reprints of this article or to subscribe to the journal, contact the AACR Publications Department at

Permissions pubs@aacr.org.

To request permission to re-use all or part of this article, contact the AACR Publications Department at permissions@aacr.org. 